\title{
A Novel Zoonotic Disease Outbreak Course to improve Surveillance and Response for the USDA
}

\author{
Julianna B. Lenoch* \\ Office of Health Informatics, Wisconsin Dept of Health Services, Madison, WI, USA
}

\section{Objective}

The United States Department of Agriculture (USDA) created a new course in 2013 to meet needs of the veterinary epidemiologists within the Veterinary Services (VS) division. The objective of this training was to provide a standard framework to investigate animal disease outbreaks, and apply practical solutions. VS has expertise in animal surveillance, this training course demonstrated how to incorporate the fundamentals of surveillance into an outbreak scenario. The goal of course was to give epidemiologist skills and practice to more quickly and efficiently conduct an investigation, allow rapid identification of a cause, apply control measures, and limit economic and health impacts of a disease.

\section{Introduction}

Outbreak Investigations course consisted to a series of 8 online modules, covering:

- Confirm that an outbreak is occurring and confirm diagnosis

- Case definition

- Descriptive Epidemiology

-Hypothesis Generation

-Analytic Epidemiology

-Preliminary control and Prevention

- Communication of findings

-Establishing disease surveillance and monitoring

The online modules were followed by live webinars, taught by the instructors who wrote each unit of the online webinar. Finally, select epidemiologists from Veterinary Services were invited to a 3-day classroom course, to collaborate with other team members and practice outbreak detection, surveillance and control. Three scenarios were created to highlight economic impact, severe consequences of an uncontrolled outbreak, OneHealth approach and the collaboration needed during a zoonotic disease outbreak.

\section{Methods}

The epidemiologists that attended were required to complete a self-assessment before and after the course to determine their comfort level, understanding of basic and advanced epidemiology processes, and ability to formulate and test hypothesis during an outbreak situation. These questions required answers in a 1-10 ordinal scale. Participants were also asked baseline information on their years of service for USDA-VS and education level.

A separate standard evaluation report was completed by the Professional Development service of VS. Participants were required to evaluate the 3-day live course within a standard questionnaire and allowed to assess 1-5 scoring in several measurement areas.

\section{Results}

Results of the pooled data from pre and post course evaluations were compared using a T-test for equality of means.

Results of the standard evaluation by Professional Development were pooled, and responses were reported with mean, median and standard deviation.
Knowledge in all nine areas improved after the training, statistically significant improvement in knowledge base was observed in five of the nine measured areas.

\section{Conclusions}

This training method was highly successful in meeting the goals of Veterinary Services, and will be offered again to selected team members. Participants valued the knowledge and expertise of the scenario facilitators, and the quality and realism of the scenarios presented. The participants appreciated the ability to work through scenarios in stages and to work in teams.

\section{Keywords}

training; surveillance; zoonotic disease; public health workforce; preparation

\section{Acknowledgments}

The USDA- VS epidemiology team at the Western Region office in Fort Collins Colorado was heavily involved in producing this outbreak investigation course. Special thanks to Brian McCluskey, Jason Lombard, Jason Baldwin, Tracey Linn and Alicia Humlicek.

\section{*Julianna B. Lenoch}

E-mail: julielenoch@gmail.com 